\title{
The Empirical Research on Public Service Motivation of Civil Servants in the Basic Level Government: Taking the Sub-district Offices in Kunming as a Case
}

\author{
Zheng Xiujuan \\ School of Public Administration, Yunnan University of Finance and Economics, Yunnan, P. R. China, \\ 650221 \\ (E-mail: 786564195@qq.com)
}

\begin{abstract}
Adopting the Perry's public service motivation scale and Coursey \& Pandys' classification method of three-dimensions, the paper investigates the public service motivation of civil servants in the basic level government in Kunming city, Yunnan province. In a word, the paper drew the following conclusion: (1) the dimension of attraction to policy making has relative high score. The administrative post has not significant influence on the dimension of attraction to policy making which is not similar to other research; (2) the dimension of commitment to the public interest and sacrifice has relative high score, but the self-sacrifice spirit of civil servants in Kunming city is not good due to the sample structure which includes $65.6 \%$ contract personnel and temporary employees; (3) the dimension of compassion has relative high score which is not corresponding to other research.
\end{abstract}

Keywords: PSM, Civil Servant, Basic Level Government, China

\section{Introduction}

Public service motivation(PSM) is a very important field for the esearch and practice of public management at present and emerged a lot of study conclusions and practical innovation based on Performance of government services, Individual job performance of civil servants and the management of civil servant through applying the theory of PSM.

\subsection{The Definition of PSM}

In 1975, Buchanan put forward the concept of PSM firstly. The Public service motivation is a kind of altruistic motives in essence. The altruistic object of public service motivation is social group, or a universal and non-discriminatory altruistic motives. From the ideal condition, public service motivation is an altruistic motive with minimal impact from the object factor. The concept of public service motivation evolved a process of constant development. At first, According to Perry and Wise (1990), PSM is defined as “an individual's predisposition to respond to motives grounded primarily or uniquely in public institutions and organizations”. Rainey and Steinbauer (1999) define it as "a general altruistic motivation to serve the interests of a community of people, a state, a nation or mankind”. Simeone (2004) defined The ideal public service motivation described as similar to the concept of love including the public service mission, strong goal and commitment, The spirit of self-sacrifice and so on. Recently, Vandenabeele, Scheepers, and Hondeghem (2006) defined it as "the belief, values and attitudes that go beyond self-interest or organizational interest, that concern the interest of a larger political entity and that induce, through public interaction, motivation for targeted action".

\subsection{The Measure Scale of PSM}

In the aspect of construct of PSM, Perry \& Wise(1990) viewed that public service motivation stems from three types of motivation, namely, emotional motive, normative motivation and rational motivation. They developed the measure scale of public service motivation further in 1996 and firstly proceeded the investigation in the MPA students. At first, Perry' scale of public service motivation was divided into six dimensions, and was confirmed as four dimensions in the later including attraction to policy making, commitment to the public interest, compassion, Self-sacrifice. So Perry's research made people understand the PSM more clear. After that, Coursey \& Pandy (2007) futher reduced the dimensions of PSM into three dimensions including attraction to policy making, commitment to the public interest, compassion.[1] Vandenabeele added the new dimension of democratic governance in his own 
research and established a scale of five dimensions with 18 items. But the most popular scale of PSM is Perry's scale of four dimensions with 24 item.

\subsection{The Research and Application of PSM in China}

The local research in China mainly emphasized the application of public service motivation theory in Chinese practice research, the specific descriptive of public service motivation dimensions in the context of Chinese culture and the effect of intermediary variables on PSM. The present research on PSM includes the concept of PSM, dimensions and scale, the differences of PSM between the public sector and private sector, the emergence and results of PSM, and the intermediary variables and so on.[2]

At present, the dimensions of attraction to policy making and compassion are the most important effect factors in the local research which is applied the PSM theory into the empirical study in China. The whole level of PSM in the context of Chinese culture is in level of middle. The PSM level of third sector is the highest, the governmental sector the second and the PSM level of male is higher than female. At the same time, some research also found that the dimensions of PSM is influenced by gender, political role, work time and unit in the context of Chinese culture.[3] However, the major difficulties for the application of PSM in China are the development of dimensions and structure of PSM, and how to adopt the structure of PSM to construct the public management talent selection mechanism, the performance promotion mechanism and the training mechanism of public service motivation. Other research also found that the dissension of compassion has not been tested in the context of Chinese culture during the development and construction of PSM dimensions and other dimensions have been tested.[4] Yan Qaing drew the conclusion that Chinese civil servants incline to patriotism, spirit needs, social values and the purpose of the party through investigating Chinese civil servants with six dimensions, but the level of four dimensions of PSM are not high. [5] Some research also come to conclusion that the deficiency of dimension of compassion is due to the PSM of Chinese civil servants is come from the performance tool which is a kind of a conscious professional roles instead of Personal emotion drive. [6]

\section{Method}

This study is based on the 14-item PSM scale. According to Kim, the APM items in Perry's (1996) scale may not be appropriate to represent the rational base of PSM. The items are not asking whether the respondents are attracted to public policy making but whether they like or dislike politics, politicians, and political phenomena. All of the scales are responded to on a 5-point Likert-type scale $(1=$ strong disagreement; 5 = strong agreement). To ensure equivalence of the measures in the Chinese versions, all the scales used in this study were translated into Chinese. To increase the accuracy of the response, each survey was distributed with a cover sheet guaranteeing anonymity.

The data for this study were collected through investing 465 civil servants who were working in the basic level government in Kunming. About 500 permanent full-time civil servants of Kunming City in Yunnan province were given surveys to complete during regular working hours; 471 surveys were returned, yielding a response rate of $94.2 \%$. Of the respondents, men were $37.4 \%$ and women were $62.6 \%$. Turning to educational background, $34.4 \%$ had at least a bachelor's degree; $65.6 \%$ had a junior college diploma. The mean of age is 37.3; About 36.1.9\% respondents had worked for more than 10 years and fewer (13.1\%) than 20 years in the civil service.

\section{Result and Analysis}

\subsection{The Descriptive for PSM}

The result shows that the whole PSM level of civil servants in the basic level government in Kunming city is relative high $(\mathrm{M}=3.93)$, Which is similar to other scholars' conclusions. The score of compassion dimension is very high $(\mathrm{M}=4.1)$, because the sample is drew from sub district offices in which the civil servants must contact with customers directly and handle a lot of complicated social events, so they have more experiences about social incidents. 


\begin{tabular}{|l|c|c|c|c|}
\hline & Minimum & Maximum & Mean & Std. Deviation \\
\hline Public service motivation & 1.50 & 6.86 & 3.9338 & .53177 \\
\hline Attraction to policy making & 1.00 & 5.00 & 3.9964 & .53805 \\
\hline Commitment to the public interest & 1.00 & 17.33 & 3.9348 & .90062 \\
\hline Compassion & 1.50 & 5.00 & 4.0871 & .50902 \\
\hline
\end{tabular}

Table 1 The descriptive for PSM

\subsection{The Dimensions of PSM}

The rational motivation is a kind of dynamic behavior based on the maximization of individual utility, the standardized motivation is a kind of dynamic behavior to conform to the norms and the emotional motivation is a kind of dynamic behavior to make emotional response to social situations.

The paper studies the PSM based on three motivation due to the following reasons: (1) rational motivation is internal mental power for individual to realize to maximize the utility of the self. Making policy is a kind of important way for individual to maximize their effectiveness instead of just for the individual ego satisfaction motivation, so as a kind of altruistic motives, individual achieves the maximization of the utility of the self means the realization of altruistic effect. We can conclude that rational motivation is the dimension of attraction to policy making. (2) Individual complies by the regulations and laws so as to as much as possible to meet the expectation of their commitment to the social role. The expectation for civil servant from society is to take up the social public duty, abide by the rules and make social commitment in the social management activities, even sacrifice individual benefits. These social expectations are social regulations for civil servants, so the standardized motivation is the dimension of commitment to the public interest and sacrifice. (3) Compassion is the only a dimension which is related to individual emotion, so it is the dimension of emotional motivation.

\subsubsection{Attraction to Policy Making (Rational Motivation)}

This dimension includes three questions, namely, (1) I am interested in making public programs that are beneficial for my country or the community I belong to; (2) Sharing my views on public policies with others is attractive to me; (3) Seeing people get benefits from the public program I have been deeply involved in brings me a great deal of satisfaction. The Mean of attraction to policy making dimension is $3.996^{[7]}$.

As for the question "I am interested in making public programs that are beneficial for my country or the community I belong to" and "Seeing people get benefits from the public program I have been deeply involved in brings me a great deal of satisfaction", the Mean are 3.94 and 4.16 respectively. The third question's mean is relative low $(\mathrm{M}=3.98)$. This result shows that the motivation level of attraction to policy making civil servants in Kunming is very high. See table 2.

\begin{tabular}{|c|c|c|c|c|c|}
\hline & Percent & Minimum & Maximum & Mean & Std. Deviation \\
\hline $\begin{array}{c}\text { 1. I am interested in making public } \\
\text { programs that are beneficial for my } \\
\text { country or the community I belong } \\
\text { to }\end{array}$ & $82.4 \%$ & 1.00 & 5.00 & 3.9419 & .70624 \\
\hline $\begin{array}{c}\text { 2. Sharing my views on public policies } \\
\text { with others is attractive to me }\end{array}$ & $78.9 \%$ & 1.00 & 5.00 & 3.8882 & .66983 \\
\hline $\begin{array}{c}\text { 3.Seeing people get benefits from the } \\
\text { public program I have been deeply } \\
\text { involved in brings me a great deal of } \\
\text { satisfaction }\end{array}$ & $89.9 \%$ & 1.00 & 5.00 & 4.1591 & .69516 \\
\hline
\end{tabular}

Table 2 the descriptive for attraction to policy making

Considering of the sample's social characteristics, the civil servants experience and understand more direct social reality and social issues due to their duty of public service, so they have more interests in public policy making. At the same time, $63.9 \%$ civil servants have a work seniority of low 10 years and an age of low 40 years old, so they concern social reform more. 
As for question 3, the percent of female for "not sure" or "disagree" is $22.3 \%$, male $18.4 \%$. as for question 3 , the female (94.2\%) have more identity than male (89.1\%), so female are concern with policy making, while male are willing to participate in policy making.

\subsubsection{Commitment to the Public Interest and}

\section{Sacrifice (Standardized Motivation)}

This dimension includes eight questions which makes self assessment test from social public interest and self-sacrifice respectively. Among questions, three questions, namely, "I consider public service my civic duty”, “To me, patriotism includes seeing to the welfare of others" and "Meaningful public service is very important to me" have higher score, 4.04, 3.99 and 3.93. The question "I am prepared to make enormous sacrifices for the good of society" has relative low score $(\mathrm{M}=3.51)$. The score of other questions all reach over 3.78. See table 3 . The result shows that the civil servants in Kunming city have low score in question "I am prepared to make enormous sacrifices for the good of society”. Maybe, the result can be explained from the sample structure which includes $65.6 \%$ contract personnel and temporary employees who have lower spirit of self-sacrifice.

\begin{tabular}{|l|c|c|c|c|c|}
\hline & Percent & Minimum & Maximum & Mean & Std. Deviation \\
\hline $\begin{array}{l}\text { 1. I am prepared to make enormous } \\
\text { sacrifices for the good of society }\end{array}$ & 56.3 & 1.00 & 5.00 & 3.5075 & .91941 \\
\hline $\begin{array}{l}\text { 2. I consider public service my civic duty } \\
\text { 3. Meaningful public service is very } \\
\text { important to me }\end{array}$ & 80.2 & 1.000 & 44.000 & 4.04086 & 2.002274 \\
\hline $\begin{array}{l}\text { 4. To me, patriotism includes seeing to } \\
\text { the welfare of others. }\end{array}$ & 80.8 & 1.00 & 5.00 & 3.9333 & .71759 \\
\hline
\end{tabular}

Table 3 the descriptive for commitment to the public interest and sacrifice

\subsubsection{Compassion (emotional motivation)}

Compassion which is evaluated from the moral level of civil servants is a recessive psychological process of exciting public servants' altruistic behavior. The dimension designs three questions which includes "It is difficult for me to contain my feelings when I see people in distress", "I feel sympathetic to the plight of the underprivileged" and "I am often reminded by daily events how dependent we are on one another". The whole score level of three questions is over 3.9 which is higher compared to other questions. See table 4.

According the correlation analysis, the result shows that the dimension of compassion is not related to the length of service and age $(\mathrm{p}=.099>.05 ; \mathrm{p}=.133>.05)$, while the gender also has no any influence on question $2(\mathrm{p}=.098>.05)$.

\begin{tabular}{|c|c|c|c|c|c|}
\hline & Percent & Minimum & Maximum & Mean & Std. Deviation \\
\hline $\begin{array}{c}\text { 1. It is difficult for me to contain my } \\
\text { feelings when I see people in distress }\end{array}$ & 81.7 & 1.00 & 5.00 & 3.9376 & .83716 \\
\hline $\begin{array}{c}\text { 2. I feel sympathetic to the plight of the } \\
\text { underprivileged }\end{array}$ & 90.7 & 1.00 & 5.00 & 4.1398 & .65310 \\
\hline $\begin{array}{l}\text { 3. I am often reminded by daily events } \\
\text { how dependent we are on one } \\
\text { another }\end{array}$ & 93.9 & 2.0 & 5.0 & 4.284 & .6340 \\
\hline
\end{tabular}

Table 4 the descriptive for Compassion

\section{Conclusion}

Through studying the PSM of civil servants from the basic governmental department in Kunming, the paper is different from the present research in the drawing way of sample. The author comes to the following conclusions: (1) the dimension of attraction to policy making has relative high score. The administrative post has not significant influence on the dimension of attraction to policy making which is not similar to other 
research. At the same time, there is significant difference in gender about the dimension of attraction to policy making; (2) the dimension of commitment to the public interest and sacrifice has relative high score, but the self-sacrifice spirit of civil servants in Kunming city is not good due to the sample structure which includes 65.6\% contract personnel and temporary employees; (3) the dimension of compassion has relative high score which is not corresponding to other research. Maybe, the result is related to the basic level of government in which the civil servants often have to contact with customers directly.

\section{References}

[1] Chunkui Zhu,Chen Wu \& Guangnan Zhu. A Review of Literature on Public Service Motivation. Journal of Public Administration, 2011(5).

[2] Ye Xianbao \& Lai Guime. Public Service Motivation: Measurement, Compassion and Influence_-Based on the Analysis of the Questionnaire Survey on Samples from Fujian Province. Chinese Public Administration, 2011(8).

[3] Yang Jingyun \& Zhang Tingjun. Public service motivation performance promotion theory and model construction. Journal of Chengdu Institute of Public Administration, 2012(1).

[4] Zhang Tingjun. An Analysis of Localization of Dimensions of Civil Servants' Public Service Motivation-Fujian-Based Research. Journal of Xidian University (Social Science Edition), 2012(3).

[5] Yan Qiang.Evaluation Study on Public Service Motivation Theory, East China Normal University Master Thesis,2010.

[6] Xiaohua Li \& Jun Dong. A Research on the Effects of Government Employees’ Public Service Motivation on Their Individual Performance. Journal of Public Administration, 2012(1).

[7] Wu Xuhong. Research on public service motivation and its antecedents. People's Tribune, 2012(23). 